\title{
Correction to: An efficient linear scheme to approximate nonlinear diffusion problems
}

\author{
Hideki Murakawa ${ }^{1}$
}

Published online: 16 January 2018

(C) The JJIAM Publishing Committee and Springer Japan KK, part of Springer Nature 2018

\section{Correction to: Japan J. Indust. Appl. Math. https://doi.org/10.1007/s13160-017-0279-3}

The original article has been published by the publisher without author's approval. The figures and citations in the publication of the original article were corrected. The corrected details are given below for your reading.

Figures 1, 2 should be replaced with Fig. 1; Figures 3, 4 should be replaced with Fig. 2; Figures 5, 6 should be replaced with Fig. 3; Figures 7, 8 should be replaced with Fig. 4; Figures 9, 10 should be replaced with Fig. 5; and Figures 11, 12 should be replaced with Fig. 6 .

The citations should be changed in the text as below.

Fig. $10 \rightarrow$ Fig. 5(b)

Fig. $1 \rightarrow$ Fig. 1(a)

The left figure of Fig. $2 \rightarrow$ Fig. 1(b)

the right figure of Fig. $2 \rightarrow$ Fig. 1(c)

Figs. 3 and $4 \rightarrow$ Fig. 2

Figs. 5 and $6 \rightarrow$ Fig. 3

Fig. $7 \rightarrow$ Fig. 4(a)

Fig. $8 \rightarrow$ Fig. 4(b)

Figs. 9 and $10 \rightarrow$ Fig. 5

Fig. $11 \rightarrow$ Fig. 6(a)

Fig. $12 \rightarrow$ Fig. 6(b)

The online version of the original article can be found under https://doi.org/10.1007/s13160-017-0279-3.

$凶$ Hideki Murakawa

murakawa@math.kyushu-u.ac.jp

1 Faculty of Mathematics, Kyushu University, 744, Motooka, Nishi-ku, Fukuoka 819-0395, Japan 
The corrected figures with captions are given in this erratum.

The original article was corrected.
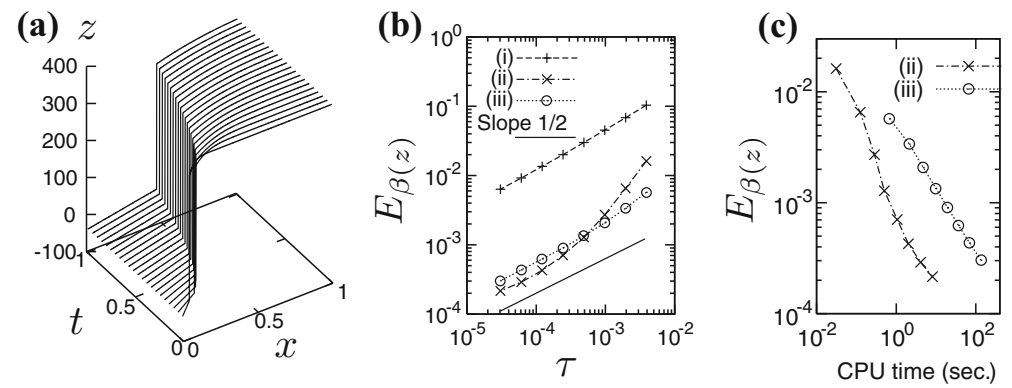

Fig. 1 a The solution of the Stefan problem (38). b, c Numerical results for the Stefan problem (38). (i) the linear scheme (3), (ii) the linear scheme (7), (iii) the nonlinear scheme (2)
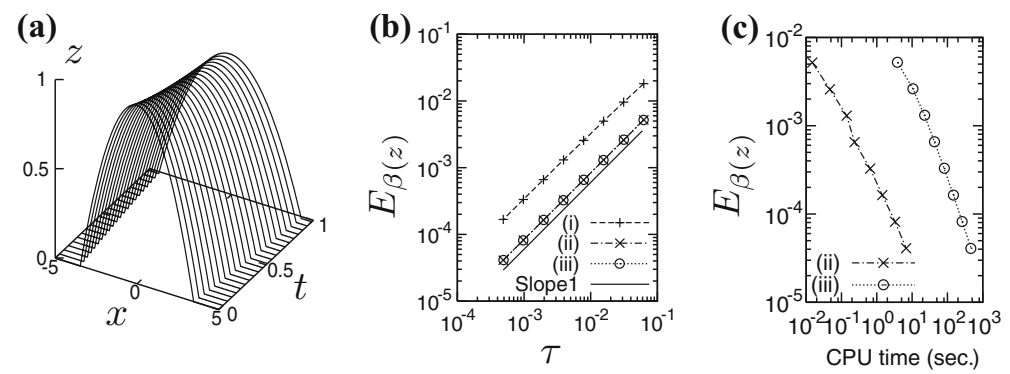

Fig. 2 a The solution of the porous medium Eq. (41) with $m=2$. b, c Numerical results for the porous medium Eq. (41) with $m=2$. (i) the linear scheme (3). (ii) the linear scheme (7). (iii) the nonlinear scheme (2)
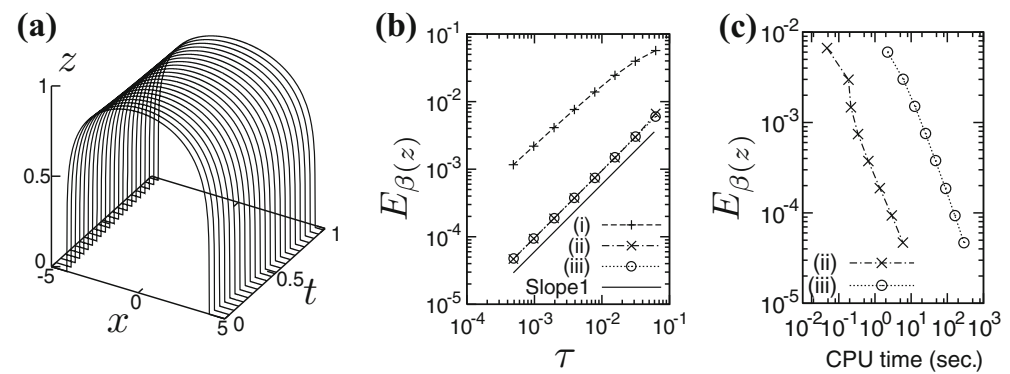

Fig. 3 a The solution of the porous medium Eq. (41) with $m=6$. b, c Numerical results for the porous medium equation (41) with $m=6$. (i) the linear scheme (3), (ii) the linear scheme (7), (iii) the nonlinear scheme (2) 
(a)

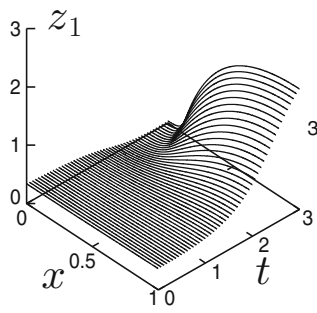

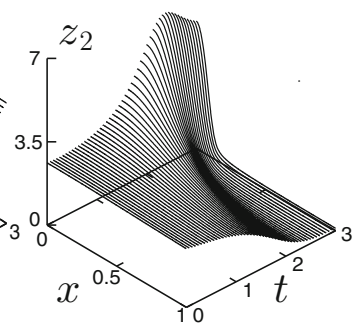

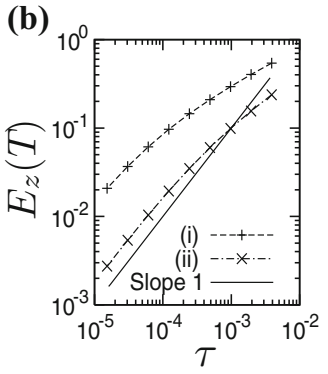

Fig. 4 a The 'solution' of (42). b Numerical results for (42). (i) the linear scheme with $\mu_{i}$ fixed, (ii) the linear scheme (7)

(a)

(b)

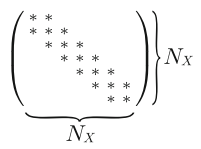

Symmetric $\times M$

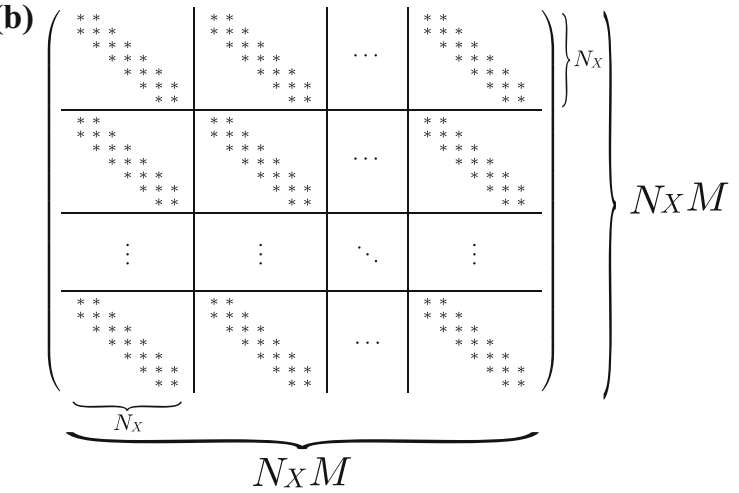

\section{Non-Symmetric \\ $\times($ Iteration Number)}

Fig. 5 a Type of matrices arising in the linear scheme (7) in one space dimension. b Type of matrices arising in the nonlinear scheme (4) in one space dimension

(a)

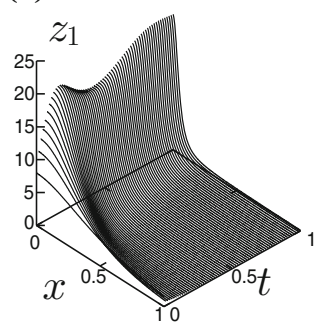

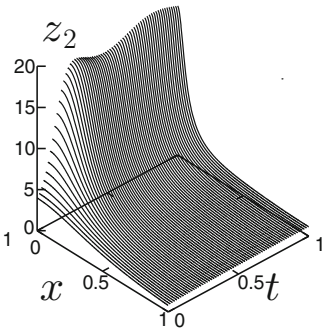

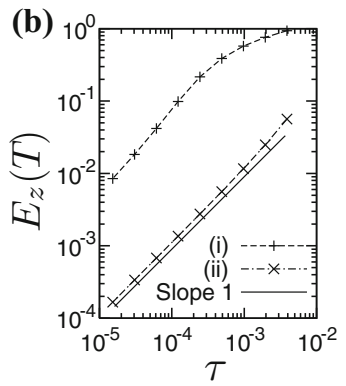

Fig. 6 a The 'solution' of (44). b Numerical results for (44). (i) the linear scheme with $\mu_{i}$ fixed, (ii) the linear scheme (7) 